\title{
A Novel Sea-Land Segmentation Algorithm Based on Local Binary Patterns for Ship Detection
}

\author{
Yu Xia, Shouhong Wan, Peiquan Jin and Lihua Yue \\ School of Computer Science and Technology \\ University of Science and Technology of China, China \\ xiay1989@mail.ustc.edu.cn,\{wansh,jpq,llyue\}@ustc.edu.cn
}

\begin{abstract}
Ship detection is an important application of optical remote sensing image processing. Sea-land segmentation is the key step in ship detection. Traditional sea-land segment methods only based on the gray-level information of an image to choose a gray threshold to segment the image; however, it is very difficult to establish a self-adapting mechanism to select a suitable threshold for different images. Thus, the segmentation result is greatly influenced by the threshold chosen for sea-land segmentation. In this paper, we are integrating the LBP feature information to propose a novel sea-land segmentation algorithm. Moreover, a new ship detection method based on our sea-land segmentation algorithm is proposed for optical remote sensing images. The performance of ship detection is measured in terms of precision and false-alarm-rate. Experimental results show that, as compared to minimum error meth$o d$, the proposed algorithm can decrease the false-alarm-rate from $23.2 \%$ to $9.24 \%$. And compared to Otsu method, the proposed algorithm improve the precision from $82.9 \%$ to $90.2 \%$.
\end{abstract}

Keywords: Ship detection, Sea-land segmentation, Optical remote sensing images, Local binary pattern

\section{Introduction}

With the rapid development of remote sensing technology, target detection of optical remote sensing images has attracted more attention because of its important role in maritime safety, maritime management, traffic monitoring, illegal smuggling, etc., [1-5] Synthetic aperture radar (SAR) images are often adopted for ship detection as they are less influenced by weather conditions and time, and they can be utilized to estimate velocities of moving targets. However, SAR images are usually with high-level speckles, insensitive to wood materials, and difficult for human interpretation. Therefore, optical satellite images have recently been employed for ship detection, as they can provide more detailed information for small target detection and ship recognition [1].

Due to the different weather conditions and illuminations, there often exist shadows, noises, fuzzy and bright spots in optical remote sensing images. Besides, the special geographical conditions of sea (such as sea wave, shoal, etc.,) and the disturbance from complex edges of sea waves and land area lead to the difficulty to detect the ship targets from the sea. The sea-land segmentation is one key issue for ship detection in optical remote sensing image, which is a typical image segmentation problem. There are two categories of sea-land segmentation methods: prior geographical information based and image analysis based methods. The former methods based on the prior geographical information of the 
detection area, using image registration to obtain the final segmentation result. But these methods are always difficult to implement because the geographical information of candidate areas are often hard to get. In optical remote sensing images, the gray-level value of targets (fore-ground) is often being considered higher than the value of the sea (background). Based on this, the latter methods mostly select a threshold to segment an image based on the graylevel histogram of this image. These methods can be applied on any un-known sea-land areas. Different from other image segmentation problems, sea-land segmentation can only depend on the gray-level information. Thus, it is difficult to choose a perfect threshold to separate the land and sea. If the threshold has been selected too small, the sea waves and other noises can be segmented as land, which will bring more false-alarms. If the threshold has been selected too big, the land edges and ship targets may be segmented as background, which will bring the problem of un-closed land and ignore some low gray-level ship targets.

Traditional image analysis based methods like minimum error method [9-10], maximum entropy [11] and Otsu [12] are most used among all optical image segmentation methods [8]. Minimum error method suppose the gray-level value distribution of objects and background is belong to Gaussian mixture model, then use one-dimension histograms to estimate the parameters and determine the final threshold value based on the minimum error standards. Maximum entropy use one-dimension histograms to calculate entropy of objects and background, then it determine the final threshold value based on the maximum entropy standard. Otsu use one-dimension histograms to calculate the variance between objects and background and obtains the final threshold value in the condition that the variance is maximized [7]. These methods only based on the gray-level histogram, but images with lots of sea waves and noises may produce more false-alarms. Besides, these methods can't be adopted to the images with even-distributed gray-level. LBP has been proved excel-lent in texture analysis and pattern recognition [6]. Calculated by comparing the gray-level between referenced pixel and its neighbors, LBP can reflect the gray-level variation patterns and protrude the edge pixels.

The local binary pattern (LBP) method was first proposed by Ojala, et al., [6]. It has been one of the most widely used descriptors for texture feature because of its resistance to lighting changes, low computational complexity, and ability to encode fine details. LBP encodes the gray-level differences between the referenced pixel of an image and its neighbors so as to fully express the texture details for the image. In the application of ship detection in optical remote sensing images, the integrity of ship targets and distinction of edge area between sea and land are very important. Comparing to the gray-level information, LBP can reflect the textural feature more comprehensively. Thus, LBP can preserve the edge information of both ship targets and junction between sea and land. The support vector machine has been proposed by Vapnik as a new kind of learning algorithm for improving traditional classification method [14-16]. Nowadays, SVM was widely used in many research fields and applications, such as target detection and recognition, fault detection, clustering, classification and so on. Many researchers have used SVM in ship detection either [17].

In this paper, we aim at improving the traditional sea-land segmentation algorithm and present a new ship detection method for optical remote sensing images. Our method firstly integrates the original gray-level remote sensing image with texture features extracted by local binary patterns (LBP) to obtain the integrated feature map (IFM). At the same time, the sea-land segmentation result can be obtained by integrating the segmentation of IFM and original gray-level together. Secondly, candidate ship targets can be extracted based on the sea-land segmentation result. Meanwhile, the geometric and texture characteristics of candidate ship targets are extracted. Finally, a classifier based on SVM is trained to justify whether the candidate ship targets are real ship targets. We conduct our experiments on 
optical remote sensing images obtained from Google-Earth to demonstrate the performance of our algorithm.

The remainder of the paper is structured as follows. Section 2 gives the algorithm of sea-land segmentation. Section 3 presents the framework of proposed ship detection algorithm. Section 4 shows our experiment results. Section 5 concludes our work and gives the directions for future studies.

\section{Sea-land Segmentation based on Local Binary Patterns}

\subsection{Local Binary Patterns}

LBP was introduced in [[5]] for texture classification. Given a referenced center pixel, LBP is computed by comparing its gray value with its neighbors, based on Formula (2.1).

$$
\begin{gathered}
L B P_{P, R}=\sum_{p=1}^{P} 2^{(p-1)} \times f_{L B P}\left(g_{c}-g_{p}\right) \\
f_{L B P}(x)= \begin{cases}1, & x \geq 0 \\
0, & \text { else }\end{cases}
\end{gathered}
$$

Where $g_{c}$ the gray-level value of the referenced pixel is, $g_{p}$ is the gray-level value of its neighbors, $P$ is the number of neighbors, and $R$ is the radius of the neighbors. LBP is a powerful texture feature descriptor, which has been widely used in texture analysis, face recognition and other applications. But LBP is very sensitive to noisy points, because the pattern value can be totally changed by a noise pixel. On the other hand, LBP is suitable to extract the edge information of noisy points. There are many new texture descriptors based on LBP have been widely used in texture analysis and image processing [18-20]. But due to the low calculation and rapid computing, LBP is more suitable for optical remote sensing image processing.

\subsection{Sea-land Segmentation}

In optical remote sensing images, there are many noisy pixels like sea waves, whose graylevel values are larger than their neighbor pixels, are often be segmented as land (foreground). Taking these pixels for example, the LBP of these pixels are always zero, according to Formula (2.1). To these real land pixels, the LBP are always not zero. Moreover, for plants or mountains in optical images, the gray-level values are lower than normal pixels, which can be segmented as sea (background). This will leads to the problem of un-closed land area, which will bring out more false-alarms. But the LBP results of these areas may be different from the sea areas. By integrating LBP extraction result and original gray image together, we can reduce the influence of noises and retain the land edge at the maximum extent. Supposing $I_{I F M}$ is the image of integrated feature map, $I_{G r a y}$ is the original gray image, $I_{L B P}$ is the LBP feature image, $x$ and $y$ is the abscissa and ordinate value of a pixel. The integrated feature map (IFM) is calculated by Formula (2.2).

$$
I_{I F M}(x, y)=\omega_{1} \times I_{G r a y}(x, y)+\omega_{2} \times I_{L B P}(x, . y)
$$

Where $\omega_{1}$ and $\omega_{2}$ are the weights to combine gray image and LBP image. After $I_{I F M}$ was calculated, a threshold $t_{I F M}$ is chosen based on Minimum Error method to segment the 
integrated LBP feature image to obtain an integrated LBP sea-land image $L S_{I F M}$. At the same time, gray sea-land image $\left(L S_{G r a y}\right)$ is obtained by segmenting $I_{\text {Gray }}$ based on the threshold $t_{\text {Gray }}$ chosen by Minimum Error method, as in Formula (2.3).

$$
\begin{aligned}
L S_{I F M}(x, y) & =\left\{\begin{array}{cc}
255, \text { if } I_{I F M}(x, y)>t_{I F M} \\
0 & \text { else }
\end{array}\right. \\
L S_{\text {Gray }}(x, y) & =\left\{\begin{array}{cc}
255, \text { if } I_{\text {Gray }}(x, y)>t_{\text {Gray }} \\
0 & \text { else }
\end{array}\right.
\end{aligned}
$$

Here, $t_{I F M}$ we choose is smaller than the exact threshold computed by Minimum Error method to retain the possible missing edge pixels and dark land pixels. After $L S_{I F M}$ and $L S_{G r a y}$ are calculated, combined sea-land image $L S_{\text {combined }}$ is finally obtained by merging $L S_{I F M}$ and $L S_{G r a y}$ together by Formula (2.4).

$$
L S_{\text {Combined }}(x, y)=L S_{I F M}(x, y) \& L S_{G r a y}(x, y)
$$

Several subsequent steps like filling black holes and clearing small areas need to be taken to get the final sea-land segmentation result $L S_{\text {Final }}$. The flowchart of proposed sea-land segmentation algorithm is shown in Figure 1. Firstly, the input optical remote sensing image is converted into gray image and the LBP feature information is integrated to obtain the integrated LBP feature image. Secondly, the integrated LBP sea-land image and gray sea-land image are obtained by threshold segmentation. Moreover, the combined sea-land image is extracted by combing the integrated LBP sea-land image and gray sea-land image together based on Formula (2.4). After these steps, the preliminary sea-land segmentation result is formed. To fill the small black holes in land area which are segmented as sea area, we find the biggest sea area (black pixels) and set other pixels as land area (white pixels). Also, small land areas whose pixels number is smaller than a threshold set in advance are set as sea areas. Based on these processing steps, the final sea-land segmentation result is obtained for ship detection in the next step.

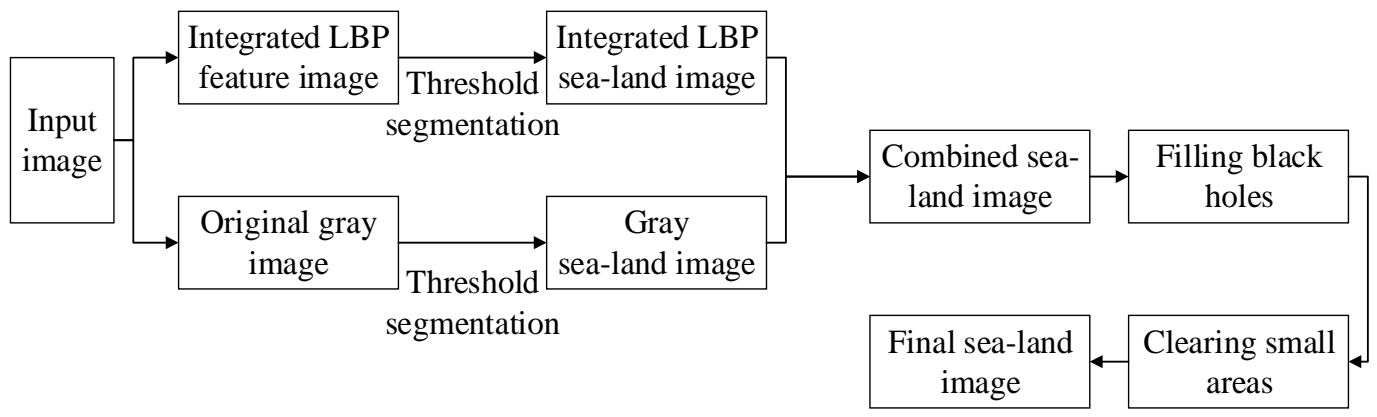

Figure 1. The Flowchart of Proposed Sea-Land Segmentation Algorithm

There are some examples are shown in Figure 2. The first column is the input optical remote sensing images and the second column are the gray images converted from the input images. The third are the combined sea-land segmentation result images from the integrated LBP sea-land images and gray sea-land images. The forth column are the black holes filling results and the last column are the final sea-land segmentation result images after clearing small areas. 


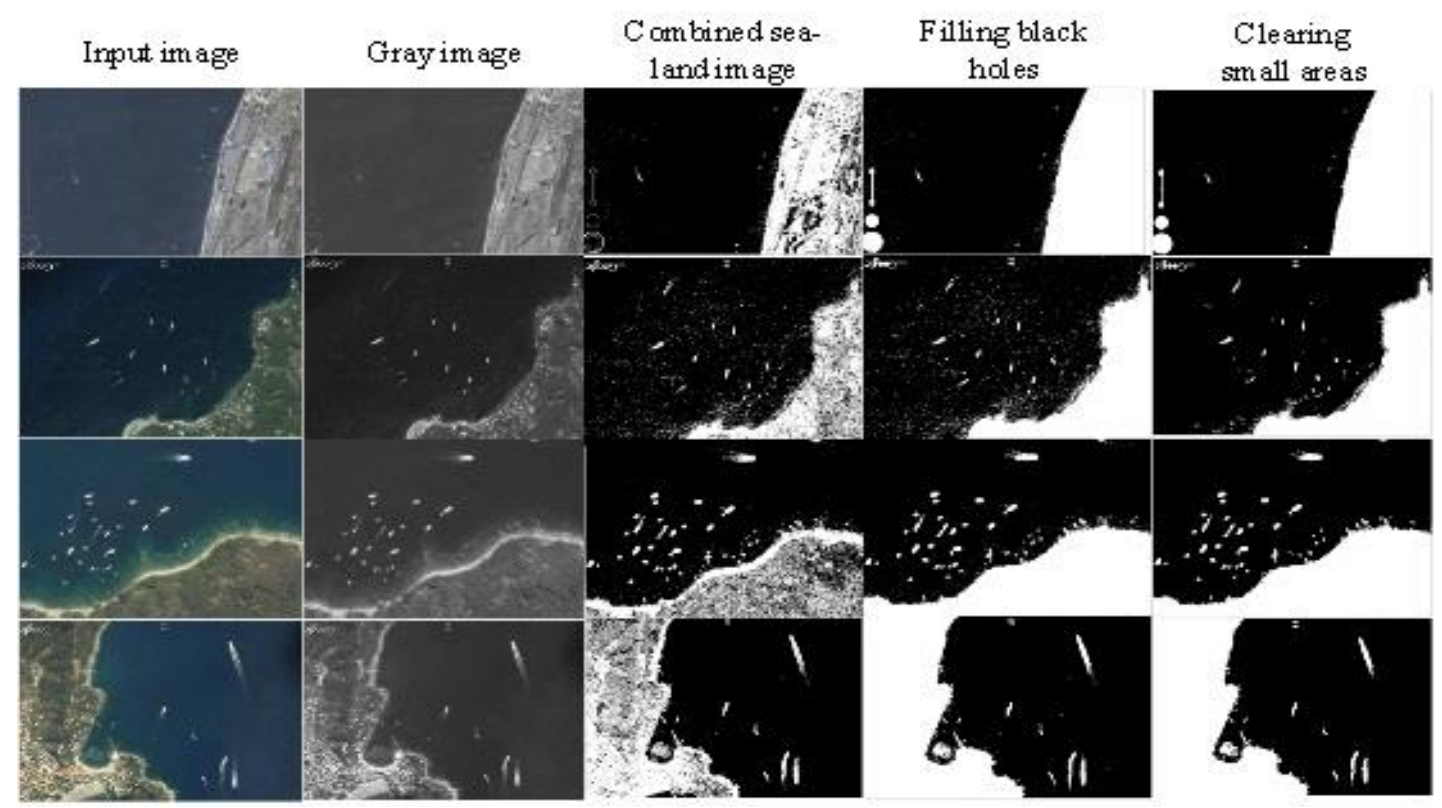

Figure 2. The Examples of Proposed Sea-Land Segmentation Results

There are some segmentation examples shown in Figure 3. $L S_{\text {Final }}$ is the segmentation result of proposed method. As we can see in row 2, the Minimum Error method segments the isolated island with the land together, while Otsu can't get the land completely. The same situation happens in row 3. In row 1, Otsu misses some four ship targets in the final segment.

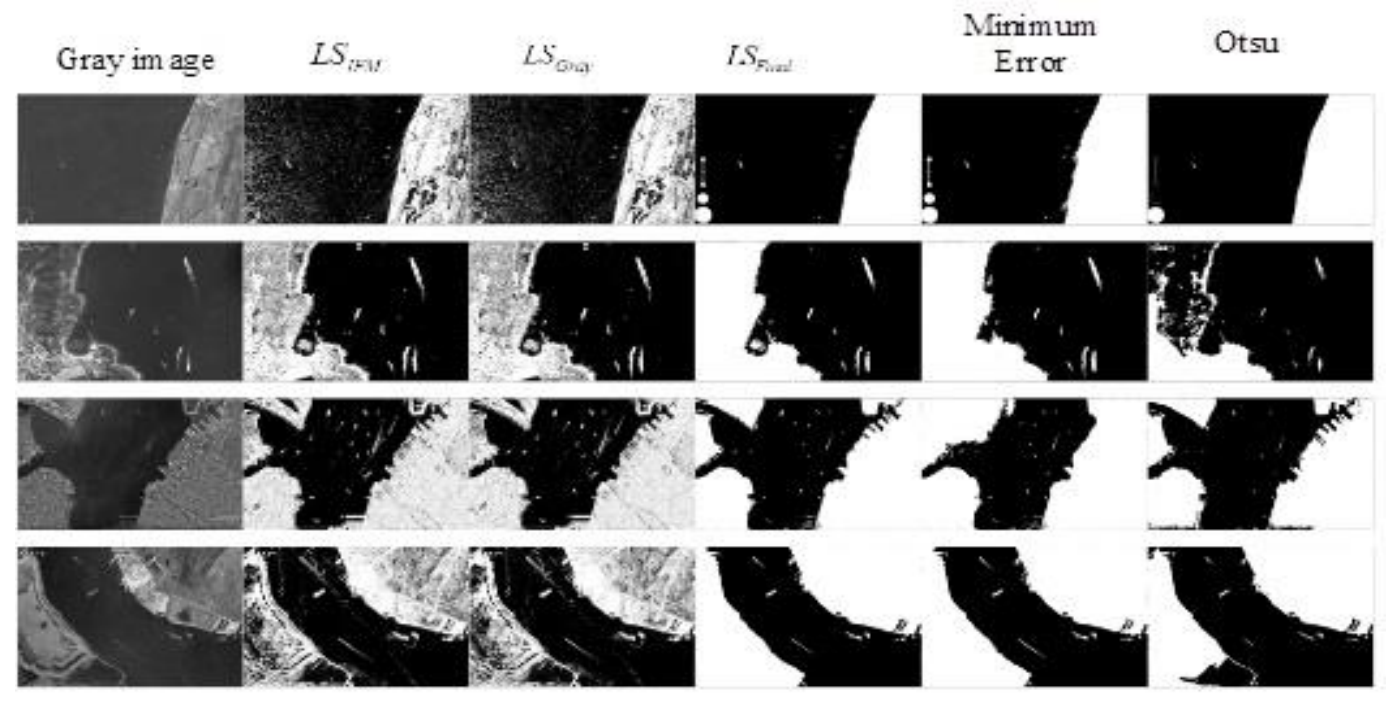

Figure 3. Sea-land Segmentation Examples

Result and Minimum Error contains some noisy edges besides the junction of land and sea. As we can see, that the proposed method performs better than minimum error method and otsu method. 


\section{Ship Detection Algorithm for Optical Remote Sensing Images}

The ship detection algorithm we proposed in shown in Algorithm 1 and Algorithm 2. Algorithm 1 refers to the training algorithm and Algorithm 2 refers to the detection algorithm. In Algorithm1, the front half part of the algorithm is the same with Algorithm 2. First we calculate the integrated feature map and the sea-land segmentation image. Secondly, candidate ship targets can be extracted based on the sea-land segmentation result. Finally, we artificially judge the candidate regions whether contains ship targets and record the samples' feature vectors to train the classifier by SVM. In this algorithm, we use many optical remote sensing images as the training samples.

Algorithm 1. Classifier training

Input: Optical remote sensing image $Q$

Output: Training classifier $S$

/* Sea-land segmentation: calculating the integrated feature map and obtaining the final sea-land segmentation image */

1: Calculating $I_{I F M}$ of $Q$ by Formula (2.2).

2: Segmenting $I_{I F M}$ and $I_{G r a y}$ to obtain $L S_{\text {Final }}$ (Formula (2.3) and Formula (2.4)).

/* Extracting candidate ship targets based on the sea-land segmentation result $* /$

3: Judging each connected region (foreground) in $L S_{F i n a l}$ and choosing the regions whose areas are smaller than the area-threshold as the candidate regions.

4: For each candidate region, using minimum error method to segment the local region of candidate area. Calculating the textural and geometric features of candidate regions.

$/ *$ Judging the candidate regions artificially and sending calculating features to train the classifier. */

5: If the candidate region contains ship targets, record the feature information and mark the sample feature vector as 1 . Go to step 7 .

6: If the candidate region contains no ship targets, record the feature information and mark the sample feature vector as 0 . Go to step 7.

7: Sending the recorded samples' feature information to SVM classifier to train the classifier $S$.

8: return the classifier $S$;

In Algorithm2, first we calculate the integrated feature map and the sea-land segmentation image. Secondly, candidate ship targets can be extracted based on the sea-land segmentation result. Finally, the training classifier is used to judge whether the candidate region contains real ship targets. The textural and geometric features we calculated in Step 4 are: the average gray value $(\overline{V G})$, the average LBP value $(\overline{V L B P})$ ratio of candidate area's height and width $(R H)$, the tightness ratio $(R T)$ and the rectangle ratio $(R R)$. They can be calculated as Formula (3.1). Supposing $I_{G r a y}$ is the gray image, $I_{L B P}$ is the LBP feature image, h, w and Sum is the 
height, width and area size of candidate region. Area is the position of the candidate region in image.

$$
\begin{aligned}
& \overline{V G}=\frac{\sum_{\forall p(i, j) \in A \text { Area }} I_{\text {Gray }}(i, j)}{(h \times w)} \\
& \overline{V L B P}=\frac{\sum_{\forall p(i, j) \in \text { Area }} I_{L B P}(i, j)}{(h \times w)} \\
& R H=h / w \\
& R T=h^{2} \times w / \text { Sum } \\
& R R=h \times w / \text { Sum }
\end{aligned}
$$

The training classifier $(S)$ is obtained by Algorithm 1, choosing the ship targets and unships targets (islands, sea waves, bright areas and land areas) artificially, using the features (in Formula (4.1)) of these targets as the training samples.

Algorithm 2. Ship Detection

Input: Optical remote sensing image $Q$

Output: Ship detection results image $R$

Preliminary: The training classifier $(S)$ to judge the candidate ship targets.

I* Sea-land segmentation: calculating the integrated feature map and obtaining the final sea-land segmentation image */

1: Calculating $I_{I F M}$ of $Q$ by Formula (2.2).

2: Segmenting $I_{I F M}$ and $I_{G \text { ray }}$ to obtain $L S_{\text {Final }}$ (Formula (2.3) and

Formula (2.4)).

/* Extracting candidate ship targets based on the sea-land segmentation result $* /$

3: Judging each connected region (foreground) in $L S_{F i n a l}$ and choosing the regions whose areas are smaller than the area-threshold as the candidate regions.

4: For each candidate region, using minimum error method to segment the local region of candidate area. Calculating the textural and geometric features of candidate regions.

$/ *$ Using the classifier to judge whether the candidate regions contain real ship targets. */

5: If the candidate region contains ship targets, record the position information and feature information, mark the ship position in $R$. Go to Step 7.

6: If the candidate region contains no ship targets, record the feature information goes to Step 7.

7: Sending the recorded feature information to SVM classifier as new training samples to update the classifier $S$.

8: return the detection result $R$; 


\section{Experiment Results}

\subsection{Experiment Setup}

In order to prove the effectiveness of proposed ship detection algorithm, experiments are implemented on the optical remote sensing images from Google-Earth. We use 50 pictures, about 400 real ship targets and 200 un-ship targets marked by human, calculating the features and sending them into the SVM classifier to train for the classifier to predict whether the candidate ship targets should be real ship targets [13]. No matter what the resolution is, our algorithm extracts them at the same resolution scale to re-process. For example, as the resolution of training image is $0.5 \mathrm{~m}$, but the image to be detected is $1.0 \mathrm{~m}$. To complete the detection process, we first use $2 * 2$ windows to extract the detection image to a new sensing image. Based on this operation, these images can be processed at the same resolution scale. The image ship detection algorithm we proposed is implemented by $\mathrm{C}++$ and OPENCV. The performance of the proposed ship detection algorithm is measured in terms of precision and false-alarmrate. For the input image $Q$, the indicators are defined as Formula (4.1) to (4.2).

$$
\begin{gathered}
\text { Precision }(Q)=\frac{N \text { umber of detected real shiptargets }}{\text { Total Number of ship targets }} \\
\text { False-Alarm - rate }(Q)=\frac{\text { Numberof detected unship targets }}{\text { Numberof detected targets }}
\end{gathered}
$$

\subsection{Experiment Results and Discussions}

We use 50 optical remote sensing images as test images, and compare our algorithm with minimum error method and otsu method. $\omega_{1}$ and $\omega_{2}$ in Formula (2.2) are both 0.5. There are some detection results shown in Figure 4. As we can see, there are more false-alarm targets detected by Minimum Error method and more ship targets missed by Otsu method.

There are 480 ship targets in all of the test images, the precision and false-alarm-rate of proposed algorithm and compared methods are shown in Table 1. As we can see, the precision of proposed algorithm (90.2\%) is about the same with Minimum Error method (90.8\%), but the false-alarm-rate of proposed algorithm $(9.24 \%)$ is much lower than Minimum Error method (23.2\%) and Otsu method (27.2\%). At the same time, the precision of proposed algorithm $(90.2 \%)$ is higher than the precision of Otsu $(82.9 \%)$. 


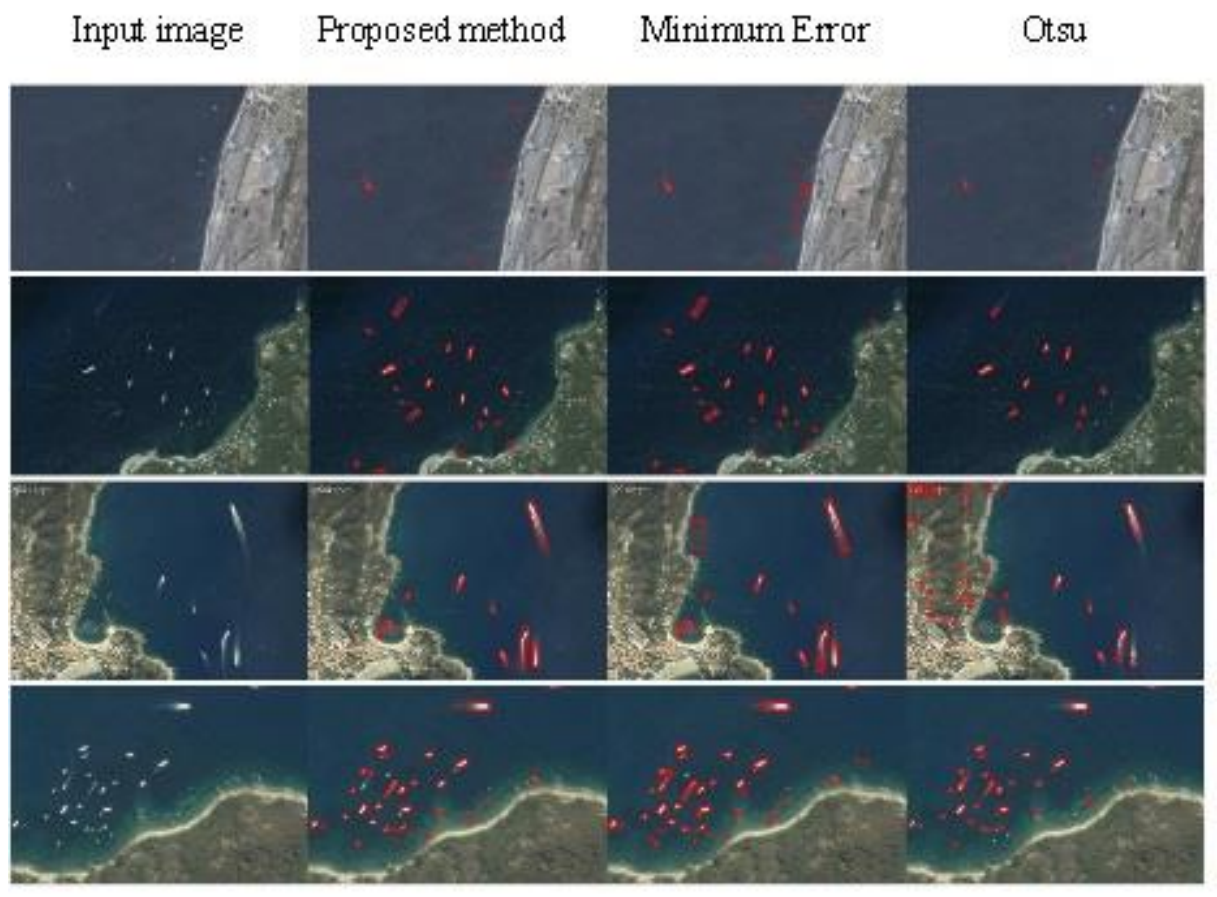

Figure 4. Samples of Ship Detection Results

Table 1. The Precision and False-Alarm-Rate Results

\begin{tabular}{|c|c|c|c|c|c|}
\hline & $\begin{array}{c}\text { Total detected } \\
\text { targets }\end{array}$ & $\begin{array}{c}\text { Correctly detected } \\
\text { ship targets }\end{array}$ & $\begin{array}{c}\text { False-alarm } \\
\text { detected targets }\end{array}$ & Precision & $\begin{array}{c}\text { False- } \\
\text { alarm rate }\end{array}$ \\
\hline $\begin{array}{c}\text { Proposed } \\
\text { method }\end{array}$ & 476 & 433 & 44 & $\begin{array}{c}432 / 480= \\
90.2 \%\end{array}$ & $\begin{array}{c}44 / 476= \\
9.24 \%\end{array}$ \\
\hline $\begin{array}{c}\text { Minimum } \\
\text { Error }\end{array}$ & 568 & 436 & 132 & $\begin{array}{c}436 / 480= \\
90.8 \%\end{array}$ & $\begin{array}{c}132 / 568= \\
23.2 \%\end{array}$ \\
\hline Otsu & 547 & 398 & 149 & $\begin{array}{c}398 / 480= \\
82.9 \%\end{array}$ & $\begin{array}{c}149 / 547= \\
27.2 \%\end{array}$ \\
\hline
\end{tabular}

\section{Conclusion}

In this paper, we present a novel sea-land segmentation algorithm based on LBP feature extraction. Moreover, a new ship detection algorithm for optical remote sensing images is proposed. Experimental results show that the proposed method can reduce the false-alarm-rate as compared to traditional methods. But the proposed method can't adopted to the optical remote sensing images with clouds. So in our future work, we will take consideration of the texture features of clouds, and analyze the difference of texture distribution between land, sea, ship targets and clouds to reduce the influence of clouds. Also, it needs more texture and geometric features to improve the precision and reduce the false-alarm-rate. 


\section{Acknowledgement}

This work is supported by the National Science Foundation of China under the grant no. 61272317 and no. 61073039.

\section{References}

[1] G. Yang, B. Li, S. Ji, F. Gao and Q. Xu, "Ship Detection From Optical Satellite Images Based on Sea Surface Analysis”, Geoscience and Remote Sensing Letters, IEEE, vol. 11, no. 3, (2014), pp. 641-645.

[2] S. Wan, P. Jin and L. Yue, "An Approach for Image Retrieval Based on Visual Saliency", International Conference on Image Analysis and Signal Processing, (IASP'09), IEEE CS, Linhai, China, (2009), pp. 172-175.

[3] L. Yachao, Z. Ruiyu, Q. Yinghui and X. Mengdao, "An Algorithm of Ship Target Detection Based on the Adaptive Background Window Function", Journal of Xi'an Jiaotong University, vol. 47, no. 6, (2013), pp. 25-30.

[4] D. Chun, S. Jixiang, L. Zhiyong and T. Shuhua, "Method for ship recognition using optical remote sensing data", Journal of Image and Graphics, vol. 17, no. 004, (2012), pp. 589-595.

[5] Z. Wei, G. Jian and H. You, "Ship detection from low observable regions in optical remote sensing imagery", Journal of Image and Graphics, vol. 17, no. 009, (2012), pp. 1181-1187.

[6] T. Ojala, M. Pietikainen and T. Maenpaa, "Multiresolution gray-scale and rotation invariant texture classification with local binary patterns", Pattern Analysis and Machine Intelligence, IEEE Transactions on, vol. 24, no. 7, (2002), pp. 971-987.

[7] Q. Wang, S. Wan and L. Yue, “A Novel Robust Algorithm for Image Segmentation”, Image and Graphics (ICIG), 2011 Sixth International Conference on. IEEE, (2011), pp. 238-243.

[8] H. Si-qi and W. Lei, "A survey of Thresholding Methods for Image Segmentation", Systems Engineering and Electronics, vol. 24, (2002), pp. 91-94.

[9] J. Kittler and J. Illingworth, "Minimum Error Thresholding", Pattern Recognition, vol. 19, (1986), pp. 41-47.

[10] S. Cho, R. Haralick and S. Yi, "Improvement of Kittler and Illingworth's Minimum Error Thresholding”, Pattern Recognition, vol. 22, (1989), pp. 609-617.

[11] J. Kapur, P. Sahop and A. Wong, "A New Method for Grey-Level Picture Thresholding Using the Entropy of Histogram”, Computer Vision Graphics, and Image Processing, vol. 29, (1985), pp. 210-239.

[12] N. A. Otsu, "Threshold Selection Method from Gray-Level Histograms", IEEE Trans. On Systems, Man and Cybernetics, vol. 13, (1983), pp. 231-235.

[13] Y. Xia, S. Wan and Yue L, "A Novel Algorithm for Ship Detection Based on Dynamic Fusion Model of Multi-feature and Support Vector Machine", Image and Graphics (ICIG), 2011 Sixth International Conference on. IEEE, (2011), pp. 521-526.

[14] V. Vapnik, "The Nature of Statistical Learning Theory”, New York: Spring-Verlag, (1995).

[15] V. Vapnik, "An overview of Statistical Learning Theory", IEEE Transactions on Neural Networks, vol. 10, no. 5, (1999), pp. 988-999.

[16] C. Cortes and V. Vapnik, "Support Vector Networks", Machine Learning, vol. 20, (1995), pp. 273-297.

[17] L. Yi and X. Shou-shi, “A New Method for Ship Target Recognition Based on Support Vector Machine", Computer Simulation, (2006), pp. 1006-9348.

[18] W.-H. Liao, "Region description using extended local ternary patterns", Pattern Recognition (ICPR), 2010 IEEE 20th International Conference, (2010), pp. 1003-1006.

[19] Z. Guo, L. Zhang and D. Zhang, "A completed modeling of local binary pattern operator for texture classification”, Image Processing, IEEE Transactions on, vol. 19, (2010), pp. 1657-1663.

[20] G. Schaefer and N. P. Doshi, "Multi-dimensional local binary pattern descriptors for improved texture analysis", In Pattern Recognition (ICPR), 2012 IEEE 21st International Conference on, (2012), pp. 2500-2503. 